\title{
Une note sur les dérivations localement nilpotentes dans les catégories monoïdales symétriques
}

\author{
Abhishek Banerjee
}

\begin{abstract}
Résumé
Dans cet article, nous construisons le morphisme exponentiel $e^{D}: A \longrightarrow A$ associé à une dérivation localement nilpotente $D$ sur un monoïde $A$ dans une catégorie monoïdale symétrique. De plus, nous montrons que le noyau de $D$ coïncide avec le sous-objet de $A$ fixé par $e^{D}$. Enfin, nous étudions comment on peut prolonger une dérivation localement nilpotente sur $A$ à sa localisation $A_{S}$, où $S \subseteq \operatorname{Hom}_{A-\operatorname{Mod}}(A, A)$ est un sous-ensemble stable par multiplication.
\end{abstract}

\begin{abstract}
In this paper, we construct the exponential morphism $e^{D}: A \longrightarrow A$ associated to a locally nilpotent derivation $D$ on a monoid $A$ in a symmetric monoidal category. Further, we show that the kernel of $D$ is identical to the subobject of $A$ invariant under $e^{D}$. Finally, we study how to extend a locally nilpotent derivation on $A$ to its localization $A_{S}$, where $S \subseteq \operatorname{Hom}_{A-\operatorname{Mod}}(A, A)$ is a multiplicatively closed subset.
\end{abstract}

\section{Introduction}

Soit $(\mathbf{C}, \otimes, 1)$ une catégorie abélienne et monoïdale symétrique vérifiant certaines hypothèses. Alors, on veut étudier les monoïdes dans la catégorie $(\mathbf{C}, \otimes, 1)$. Par exemple, dans [15], Vitale a développé une théorie de Morita pour les monoïdes

Received by the editors in August 2015.

Communicated by S. Caenepeel.

2010 Mathematics Subject Classification : 13N15. 
dans $(\mathbf{C}, \otimes, 1)$. Quand la catégorie abélienne est également une catégorie modèle, la théorie de monoïdes dans $\mathbf{C}$ a été étudiée par Hovey [12]. Dans, [2], nous avons étudié l'algèbre de Lie qui est constituée des dérivations sur un monoïde dans $(\mathbf{C}, \otimes, 1)$. En outre, nombreux auteurs ont étudié la géométrie algébrique sur une catégorie monoïdale symétrique (voir, par exemple, [7], [11], [14]) en utilisant les monoïdes dans $(\mathbf{C}, \otimes, 1)$. Donc, il est naturel de se demander s'il existe un analogue de la géométrie algébrique affine pour les categories monoïdales symétriques. Pour développer la géométrie algébrique affine, il faut développer la théorie des dérivations localement nilpotentes (voir, par exemple, [10]) pour les monoïdes dans une catégorie monoïdale symétrique. Cet article est une première étape dans cette direction. Pour savoir plus sur les dérivations localement nilpotentes, voir, par exemple, [4], [5], [6], [9].

Soit $(\mathbf{C}, \otimes, 1)$ une catégorie monoïdale symétrique. Supposons que $\mathbf{C}$ est abélienne et $\mathbf{C}$ satisfait quelques conditions détaillées dans Section 2. Étant donné un monoïde $A$ dans $\mathbf{C}$, nous commençons par définir les dérivations localement nilpotentes $D: A \longrightarrow A$ sur $A$ (voir Définition 2.1). Ensuite, nous construisons le morphisme exponentiel $e^{D}: A \longrightarrow A$ associé à une derivation localement nilpotente $D$. De plus, nous montrons que $e^{D}$ est un automorphisme des monoïdes et le sous-groupe des automorphismes de $A$ engendré par les morphismes exponentiels est normal. Enfin, nous montrons que les sous-objet invariant $A^{D}:=\operatorname{Ker}\left(e^{D}-1\right)$ de $D$ coïncide avec le noyau $\operatorname{Ker}(D)$ de $D$.

Dans la deuxième partie de cet article, nous supposons que $1 \in \mathbf{C}$ est un objet de type fini dans la catégorie $C$. Par exemple, soit $X$ un espace topologique compact et soit $\mathcal{B}$ une base de $X$ telle que chaque ouvert $U \in \mathcal{B}$ est compact. Alors, si $\mathcal{A}$ est un faisceau d'anneaux commutatifs sur $X, \mathcal{A}$ est un objet de type fini dans la catégorie $\mathcal{A}-\operatorname{Mod}$ des $\mathcal{A}$-modules. Nous considérons un monoïde commutatif $A$ dans $C$ et $S \subseteq \operatorname{Hom}_{A-\operatorname{Mod}}(A, A)$ un sous-ensemble stable par multiplication (composition). Nous montrons qu'une dérivation $D: A \longrightarrow A$ induit une dérivation $\mathcal{E}(D)$ sur l'anneau commutatif $\mathcal{E}(A):=\operatorname{Hom}_{A-\operatorname{Mod}}(A, A)$. Puis, nous considérons le monoïde localisé $A_{S}$ au sens de [1, § 3]. Alors, pour une dérivation localement nilpotente $D: A \longrightarrow A$, nous étudions comme on peut prolonger $D$ à une dérivation localement nilpotente $D_{S}$ sur le monoïde localisé $A_{S}$. Quand $A$ est un monoïde intègre (voir Definition 3.3) et le prolongement $D_{S}: A_{S} \longrightarrow A_{S}$ de $D$ est une dérivation localement nilpotente, nous montrons que $S \subseteq \operatorname{Ker}(\mathcal{E}(D))$. Inversement, si $S \subseteq \mathcal{E}(A)$ est un sous-ensemble stable par multiplication contenu dans le noyau $\operatorname{Ker}(\mathcal{E}(D))$, nous montrons que $D_{S}$ est une dérivation localement nilpotente sur $A_{S}$.

\section{Dérivations localement nilpotentes}

Soit $k$ un corps de caractéristique nulle. Soit $(\mathbf{C}, \otimes, 1)$ une catégorie monoïdale symétrique, abélienne et $k$-linéaire. De plus, supposons que les limites inductives dans $\mathbf{C}$ commutent avec $\otimes$ et les limites inductives filtrantes dans $\mathbf{C}$ commutent aux limites projectives finies. Pour chaque monoïde $A$ dans $\mathbf{C}$, notons par $m_{A}: A \otimes A \longrightarrow A$ la multiplication sur $A$ et notons par $e_{A}: 1 \longrightarrow A$ le morphisme unitaire sur $A$. Dans la suite, tous les monoïdes sont commutatifs. 
Définition 2.1. (voir [3]) Soit $A$ un monö̈de dans $(\mathbf{C}, \otimes, 1)$. Un morphisme $D: A \longrightarrow$ A est appelé une dérivation sur $A$ si:

$$
D \circ m_{A}=m_{A} \circ(D \otimes 1+1 \otimes D): A \otimes A \longrightarrow A
$$

Soit $A$ un monoïde et $D: A \longrightarrow A$ une dérivation sur $A$. Puisque $C$ est abélienne, il existe un noyau $\operatorname{Ker}(D)$ de $D$. Considérons la suite croissante des sous-objets de $A$ :

$$
\operatorname{Ker}(D) \subseteq \operatorname{Ker}\left(D^{2}\right) \subseteq \operatorname{Ker}\left(D^{3}\right) \subseteq \ldots
$$

La dérivation $D$ est appelée localement nilpotente si:

$$
\underset{i \geq 1}{\lim _{i \rightarrow}} \operatorname{Ker}\left(D^{i}\right)=A
$$

où $\lim \operatorname{Ker}\left(D^{i}\right)$ est la limite directe des objets $\operatorname{Ker}\left(D^{i}\right), i \geq 1$. On va noter par $\overrightarrow{i \geq 1}$

$D L N(A)$ l'ensemble des dérivations localement nilpotentes sur $A$. On peut définir maintenant le morphisme exponentiel $e^{D}: A \longrightarrow A$ correspondant à une dérivation localement nilpotente.

Proposition 2.2. Soit $A$ un monoïde et $D: A \longrightarrow A$ une dérivation localement nilpotente sur $A$. Alors, il existe un morphisme exponentiel $e^{D}: A \longrightarrow A$. De plus, $e^{D}$ est un morphisme des monoïdes dans $(\mathbf{C}, \otimes, 1)$.

Proof. Considérons les morphismes

$$
\sum_{j=0}^{i-1} \frac{D^{j}}{j !}: \operatorname{Ker}\left(D^{i}\right) \longrightarrow A, \quad i \geq 1
$$

qui induisent le morphisme exponentiel

$$
e^{D}:=\sum_{j=0}^{\infty} \frac{D^{j}}{j !}: \underset{i \geq 1}{\lim } \operatorname{Ker}\left(D^{i}\right)=A \longrightarrow A
$$

Puisque $D$ est une dérivation, on peut vérifier que

$$
\frac{D^{k}}{k !} \circ m_{A}=m_{A} \circ\left(\sum_{i=0}^{k} \frac{D^{i}}{i !} \otimes \frac{D^{k-i}}{(k-i) !}\right), \quad \forall k \geq 0
$$

Pour $i, j$ fixés, on a

$$
m_{A} \circ\left(e^{D} \otimes e^{D}\right)=m_{A} \circ\left(\sum_{m=0}^{i-1} \frac{D^{m}}{m !} \otimes \sum_{n=0}^{j-1} \frac{D^{n}}{n !}\right): \operatorname{Ker}\left(D^{i}\right) \otimes \operatorname{Ker}\left(D^{j}\right) \longrightarrow A
$$

En appliquant (2.6), on peut écrire le morphisme $m_{A} \circ\left(e^{D} \otimes e^{D}\right): \operatorname{Ker}\left(D^{i}\right) \otimes$ $\operatorname{Ker}\left(D^{j}\right) \longrightarrow A$ comme:

$$
m_{A} \circ\left(\sum_{m=0}^{i-1} \frac{D^{m}}{m !} \otimes \sum_{n=0}^{j-1} \frac{D^{n}}{n !}\right)=m_{A} \circ\left(\sum_{m=0}^{\infty} \frac{D^{m}}{m !} \otimes \sum_{n=0}^{\infty} \frac{D^{n}}{n !}\right)=\sum_{k=0}^{\infty} \frac{D^{k}}{k !} \circ m_{A}=e^{D} \circ m_{A}
$$


Ainsi, pour $i, j$ fixés, on a

$$
m_{A} \circ\left(e^{D} \otimes e^{D}\right)=e^{D} \circ m_{A}: \operatorname{Ker}\left(D^{i}\right) \otimes \operatorname{Ker}\left(D^{j}\right) \longrightarrow A
$$

Donc, on a

$$
m_{A} \circ\left(e^{D} \otimes e^{D}\right)=e^{D} \circ m_{A}: \underset{i, j \geq 1}{\lim } \operatorname{Ker}\left(D^{i}\right) \otimes \operatorname{Ker}\left(D^{j}\right)=A \otimes A \longrightarrow A
$$

Proposition 2.3. Soit $A$ un monoüde et $D: A \longrightarrow A$ une dérivation localement nilpotente sur $A$. Notons par $A$ ut $(A)$ le groupe des automorphismes de monoïde $A$. Alors, $e^{D} \in A u t(A)$. De plus, le sous-groupe de Aut $(A)$ engendré par les éléments $\left\{e^{D} \mid D \in D L N(A)\right\}$ est normal.

Proof. Puisque $-D$ est une dérivation localement nilpotente, on peut définir $e^{-D}$ : $A \longrightarrow A$. Pour tous les entiers positifs $i, j$, le sous-objet $\operatorname{Ker}\left(D^{i}\right)$ est invariant par $D^{j}$. Ainsi, chaque sous-objet $\operatorname{Ker}\left(D^{i}\right), i \geq 1$, est invariant par $e^{D}$ et $e^{-D}$. Pour $i$ fixé, considérons les restrictions de $e^{D}$ et $e^{-D}$ à $\operatorname{Ker}\left(D^{i}\right)$. Alors, on a

$$
\left(e^{D} \circ e^{-D}\right)\left|\operatorname{Ker}\left(D^{i}\right)=\left(\left(\sum_{m=0}^{i-1} \frac{D^{m}}{m !}\right) \circ\left(\sum_{n=0}^{i-1} \frac{(-D)^{n}}{n !}\right)\right)\right| \operatorname{Ker}\left(D^{i}\right)=1
$$

En passant à la limite directe, on a $e^{D} \circ e^{-D}=1$ sur $A$. De même, on a $e^{-D} \circ e^{D}=$ 1 sur $A$. Donc, $e^{D}: A \longrightarrow A$ est un automorphisme. Si $\alpha \in A u t(A), \alpha D \alpha^{-1}$ est également une dérivation localement nilpotente. Considérons les morphismes

$$
\sum_{j=0}^{i-1} \frac{\left(\alpha D \alpha^{-1}\right)^{j}}{j !}=\alpha\left(\sum_{j=0}^{i-1} \frac{D^{j}}{j !}\right) \alpha^{-1}: \operatorname{Ker}\left(\left(\alpha D \alpha^{-1}\right)^{i}\right) \stackrel{\alpha^{-1}}{\longrightarrow} \operatorname{Ker}\left(D^{i}\right) \stackrel{\sum_{j=0}^{i-1} \frac{D^{j}}{j !}}{\longrightarrow} A \stackrel{\alpha}{\longrightarrow} A
$$

En passant à la limite inductive, on a $e^{\alpha D \alpha^{-1}}=\alpha e^{D} \alpha^{-1}$. Ainsi, le sous-groupe de $A u t(A)$ engendré par les éléments $\left\{e^{D} \mid D \in D L N(A)\right\}$ est normal.

Désormais, on pose $A^{D}=\operatorname{Ker}\left(e^{D}-1\right)$. Autrement dit, $A^{D}$ est le sous-objet des invariants de $e^{D}$. Le résultat suivant va permettre de décrire l'objet $A^{D}$.

Proposition 2.4. Soit $A$ un monoïde et $D: A \longrightarrow A$ une dérivation localement nilpotente sur A. Alors, $A^{D}=\operatorname{Ker}(D)$.

Proof. Fixons un entier $i>0$ et notons par $D_{i}$ la restriction de $D$ à $\operatorname{Ker}\left(D^{i}\right)$. On a

$$
\operatorname{Ker}\left(\left(e^{D}-1\right) \mid \operatorname{Ker}\left(D^{i}\right)\right)=\operatorname{Ker}\left(\sum_{m=1}^{i-1} \frac{D_{i}^{m}}{m !}\right)
$$

En vertu de la définition du noyau d'un morphisme, pour tous les objets $B$ dans C:

$$
\operatorname{Hom}\left(B, \operatorname{Ker}\left(\sum_{m=1}^{i-1} \frac{D_{i}^{m}}{m !}\right)\right)=\left\{h \in \operatorname{Hom}(B, A) \mid D^{i} h=0 \& \sum_{m=1}^{i-1} \frac{D^{m} h}{m !}=0\right\}
$$


Si $h \in \operatorname{Hom}(B, A)$ tel que $D^{i} h=0$ et $\sum_{m=1}^{i-1} \frac{D^{m} h}{m !}=0$, on a

$$
D^{i-1} h=D^{i-2}\left(\sum_{m=1}^{i-1} \frac{D^{m} h}{m !}\right)=\sum_{m=1}^{i-1} \frac{D^{m+i-2} h}{m !}=0 \Rightarrow \sum_{m=1}^{i-2} \frac{D^{m} h}{m !}=\sum_{m=1}^{i-1} \frac{D^{m} h}{m !}=0
$$

Par induction, $D h=0$. Inversement, si $D h=0$, on a $D^{i} h=0$ et $\sum_{m=1}^{i-1} \frac{D^{m} h}{m !}=0$. Alors,

$$
\operatorname{Hom}\left(B, \operatorname{Ker}\left(\sum_{m=1}^{i-1} \frac{D_{i}^{m}}{m !}\right)\right)=\{h \in \operatorname{Hom}(B, A) \mid D h=0\}=\operatorname{Hom}\left(B, \operatorname{Ker}\left(D_{i}\right)\right)
$$

En utilisant le lemme de Yoneda et (2.13), on a $\operatorname{Ker}\left(\left(e^{D}-1\right) \mid \operatorname{Ker}\left(D^{i}\right)\right)=\operatorname{Ker}\left(D_{i}\right)$. En vertu de la définition d'un noyau dans une catégorie abélienne, on peut écrire

$$
\begin{gathered}
\operatorname{Ker}\left(\left(e^{D}-1\right) \mid \operatorname{Ker}\left(D^{i}\right)\right)=\lim \left(0 \longrightarrow \operatorname{Ker}\left(D^{i}\right) \stackrel{e^{D}-1}{\longleftarrow} \operatorname{Ker}\left(D^{i}\right)\right) \\
\operatorname{Ker}\left(D_{i}\right)=\lim \left(0 \longrightarrow \operatorname{Ker}\left(D^{i}\right) \stackrel{D_{i}}{\longleftarrow} \operatorname{Ker}\left(D^{i}\right)\right)
\end{gathered}
$$

Rappelons que les limites inductives filtrantes commutent avec les limites projectives finies. Puisque $A$ est la limite inductive filtrante $\operatorname{des} \operatorname{Ker}\left(D^{i}\right), i>0$, on a

$$
\operatorname{Ker}\left(e^{D}-1\right)=\stackrel{\lim }{\longrightarrow} \operatorname{Ker}\left(\left(e^{D}-1\right) \mid \operatorname{Ker}\left(D^{i}\right)\right)=\stackrel{\lim }{\longrightarrow} \operatorname{Ker}\left(D_{i}\right)=\operatorname{Ker}(D)
$$

\section{Localisation et dérivations localement nilpotentes}

Soit $(\mathbf{C}, \otimes, 1)$ une catégorie monoïdale symétrique comme dans Section 2 et soit $A$ un monoïde commutatif dans $(\mathbf{C}, \otimes, 1)$. Alors, il s'ensuit que $\mathcal{E}(A):=$ $\operatorname{Hom}_{A-\operatorname{Mod}}(A, A)$ est un anneau commutatif (voir Eckmann-Hilton [8]). Si $S \subseteq \mathcal{E}(A)$ est un sous-ensemble stable par multiplication, nous considérons le monoïde localisé $A_{S}$ comme dans $[1, \S 3]$. Nous étudions maintenant comme on peut prolonger une dérivation $D \in D L N(A)$ à une dérivation localement nilpotente $D_{S}$ sur le monoïde localisé $A_{S}$.

Dans cette section, nous supposons que 1 est un objet de type fini dans la catégorie C. Autrement dit, le foncteur $\operatorname{Hom}_{\mathbf{C}}(1, \ldots)$ commute aux colimites filtrantes des monomorphismes. Par exemple, supposons que $X$ est un espace topologique compact et $\mathcal{B}$ est une base de $X$ telle que chaque ouvert $U \in \mathcal{B}$ est compact. Alors, si $\mathcal{A}$ est un faisceau d'anneaux commutatifs sur $X, \mathcal{A}$ est un objet de type fini dans la catégorie monoïdale symétrique des $\mathcal{A}$-modules (voir [13, Corollary 3.4]) De plus, nous supposons que les colimites filtrantes dans $\mathbf{C}$ commutent aux limites finies.

Étant donnée une dérivation $D: A \longrightarrow A$ au sens de Définition 2.1, nous commençons en montrant qu'on dispose d'une dérivation $\mathcal{E}(D)$ sur $\mathcal{E}(A)$ correspondant à $D$. Puisque $\operatorname{Hom}_{\mathrm{C}}(1, A) \cong \operatorname{Hom}_{A-\operatorname{Mod}}(A, A)=\mathcal{E}(A)$, pour chaque $s \in \mathcal{E}(A)$, on a un élément de $\operatorname{Hom}_{\mathrm{C}}(1, A)$ qui sera noté par $s^{\prime}: 1 \longrightarrow A$. En composant avec $D: A \longrightarrow A$, on obtient $D \circ s^{\prime} \in \operatorname{Hom}_{\mathbf{C}}(1, A) \cong \operatorname{Hom}_{A-\operatorname{Mod}}(A, A)$. 
Alors, $D$ induit un morphisme $\mathcal{E}(D): \operatorname{Hom}_{A-\operatorname{Mod}}(A, A) \longrightarrow \operatorname{Hom}_{A-\operatorname{Mod}}(A, A)$. Notre premier résultat est le suivant:

Proposition 3.1. Soit $A$ un monoïde commutatif dans $(\mathbf{C}, \otimes, 1)$ et soit $D: A \longrightarrow$ $A$ une dérivation. Alors, $\mathcal{E}(D): \mathcal{E}(A) \longrightarrow \mathcal{E}(A)$ est une dérivation sur l'anneau $\mathcal{E}(A)$. De plus, si $D$ est une dérivation localement nilpotente, $\mathcal{E}(D)$ est une dérivation localement nilpotente sur $\mathcal{E}(A)$.

Proof. Prenons $s, t \in \mathcal{E}(A)$ et considérons $s^{\prime}, t^{\prime}: 1 \longrightarrow A$ correspondant respectivement à $s, t$ comme ci-dessus. Alors, on a un diagramme commutatif:

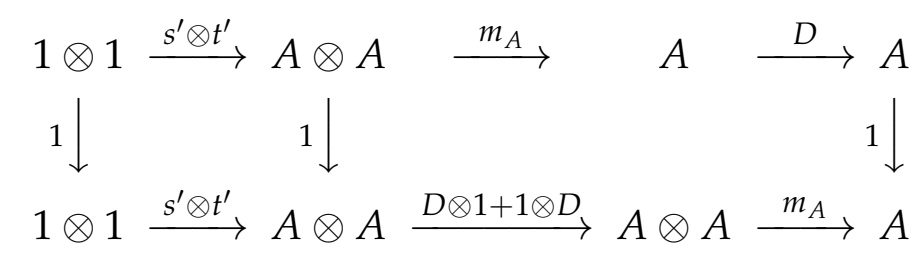

Le morphisme du haut du diagramme (3.1) correspond à $\mathcal{E}(D)(s t) \in \mathcal{E}(A)$ et le morphisme du bas correspond à $(\mathcal{E}(D)(s)) \circ t+s \circ(\mathcal{E}(D)(t)) \in \mathcal{E}(A)$. Il résulte que $\mathcal{E}(D)$ est une dérivation sur $\mathcal{E}(A)$.

Supposons maintenant que $D$ est une dérivation localement nilpotente. Alors, nous voyons qu'on a:

$$
\begin{aligned}
\operatorname{Hom}_{A-\operatorname{Mod}}(A, A) \cong & \operatorname{Hom}_{\mathbf{C}}(1, A) \cong \\
& \operatorname{Hom}_{\mathbf{C}}\left(1, \operatorname{colim}_{i \geq 1} \operatorname{Ker}\left(D^{i}\right)\right) \cong \underset{i \geq 1}{\operatorname{colim}} \operatorname{Hom}_{\mathbf{C}}\left(1, \operatorname{Ker}\left(D^{i}\right)\right)
\end{aligned}
$$

(rappelons que $1 \in \mathbf{C}$ est un objet de type fini). Alors, pour chaque $s \in \mathcal{E}(A)$, il existe un entier $i_{0} \geq 1$ tel que $\operatorname{Im}\left(s^{\prime}: 1 \longrightarrow A\right)$ est un sous-objet de $\operatorname{Ker}\left(D^{i_{0}}\right)$. Alors, $D^{i_{0}} \circ s^{\prime}=0$ et donc $(\mathcal{E}(D))^{i_{0}}(s)=0$. Il résulte que $\mathcal{E}(D)$ est une dérivation localement nilpotente sur $\mathcal{E}(A)$.

Nous rappelons maintenant la localisation d'un monoïde commutatif étudiée dans $[1, \S 3]$. Si $s \in \mathcal{E}(A)$ est un élément, on pose:

$$
A_{s}:=\operatorname{colim}(A \stackrel{s}{\longrightarrow} A \stackrel{s}{\longrightarrow} \ldots)
$$

Plus généralement, soit $S \subseteq \mathcal{E}(A)$ un sous-ensemble stable par multiplication tel que $1 \in S$. Alors, on obtient un morphisme $A_{s} \longrightarrow A_{\text {st }}$ pour chaque $s, t \in \mathcal{E}(A)$. On pose:

$$
A_{S}:=\underset{s \in S}{\operatorname{colim}} A_{S}
$$

Pour savoir plus sur la localisation des monoïdes, voir [1]. En particulier, chaque $s \in S \subseteq \mathcal{E}(A)$ induit un isomorphisme $s: A_{s} \cong A_{s}$. Nous montrons qu'on peut prolonger une dérivation $D$ sur $A$ à une dérivation $D_{S}$ sur $A_{S}$.

Proposition 3.2. Soit $A$ un monö̈de commutatif dans $(\mathbf{C}, \otimes, 1)$ et soit $S \subseteq \mathcal{E}(A)$ un sous-ensemble stable par multiplication. Soit $D$ une dérivation sur $A$. Alors, on peut prolonger $D$ à une dérivation $D_{S}$ sur la localisation $A_{S}$. 
Proof. Pour $s \in \mathcal{E}(A)$, nous considérons le diagramme commutatif suivant:

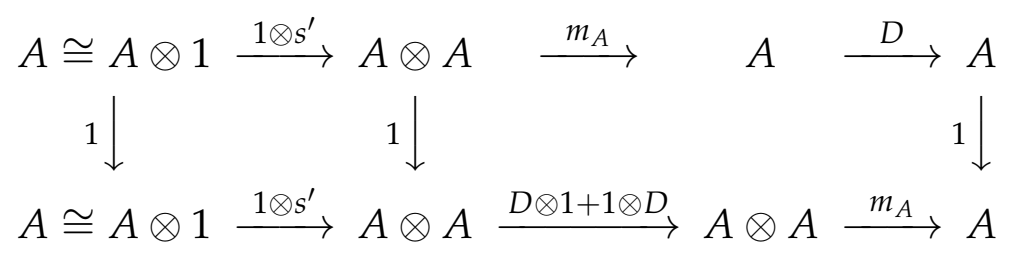

Il suit du diagramme (3.5) qu'on a:

$$
D \circ s=s \circ D+\mathcal{E}(D)(s)
$$

Puisque $s$ induit un isomorphisme $s: A_{s} \stackrel{\cong}{\longrightarrow} A_{s}$, l'égalité dans (3.6) nous donne un carré commutatif:

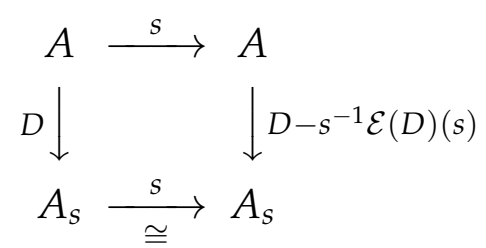

et plus généralement, un diagramme commutatif:

$$
\begin{gathered}
A \stackrel{s}{\longrightarrow} A \stackrel{s}{\longrightarrow} A \stackrel{s}{\longrightarrow} A \stackrel{s}{\longrightarrow} \ldots \\
D \downarrow D-\frac{\mathcal{E}(D)(s)}{s} \downarrow D-2 \frac{\mathcal{E}(D)(s)}{s} \downarrow D-3 \frac{\mathcal{E}(D)(s)}{s} \downarrow \\
A_{S} \stackrel{s}{\longrightarrow} A_{s} \stackrel{s}{\cong} A_{s} \stackrel{s}{\cong} A_{s} \stackrel{s}{\longrightarrow} \ldots
\end{gathered}
$$

En général, étant donné un système filtrant $\left\{M_{i}\right\}_{i \in I}$ des $A$-modules, nous considérons sa colimite $M$ dans la catégorie $C$. Alors, il est clair que $M$ est muni d'une structure d'un $A$-module et donc $M$ est aussi la colimite du système $\left\{M_{i}\right\}_{i \in I}$ dans $A-\operatorname{Mod}$. En particulier, notons que la colimite du système $(A \stackrel{s}{\longrightarrow} A \stackrel{s}{\longrightarrow} \ldots)$ dans la catégorie $A-\operatorname{Mod}$ coïncide avec sa colimite dans $\mathbf{C}$. Alors, en prenant la colimite filtrante dans (3.8), on a un prolongement $D_{s}: A_{S} \longrightarrow A_{S}$ de $D$. De plus, $S$ étant stable par multiplication, on a un prolongement $D_{S}: A_{S}=\underset{S \in S}{\operatorname{colim}} A_{S} \longrightarrow$ colim $A_{S}=A_{S}$ de $D$. On peut vérifier aisément que $D_{S}$ est une dérivation sur $A_{S} \in S$

Définition 3.3. Soit $A$ un monö̈de commutatif dans $(\mathbf{C}, \otimes, 1)$. Nous disons que A est intègre si tous les éléments non nuls de $\mathcal{E}(A)=\operatorname{Hom}_{A-\operatorname{Mod}}(A, A)$ sont des monomorphismes. En particulier, si $A$ est intègre, $\mathcal{E}(A)$ est un anneau intègre.

Lemme 3.4. Soit $R$ une $k$-algèbre, où $k$ est un corps de caractéristique nulle. Supposons que $R$ est un anneau intègre et soit $d: R \longrightarrow R$ une dérivation localement nilpotente et k-linéaire. Alors, si $u \in R$ est un élément inversible dans $R$, on a $d(u)=0$. 
Proof. Puisque $u$ est inversible, nous prenons $v \in R$ tel que $u v=1$. On sait que $d(1)=0$ et donc $d^{N}(1)=0$ pour chaque $N \geq 1$. Soit $m \geq 0$ (resp. $n \geq 0$ ) le plus grand entier tel que $d^{m}(u) \neq 0$ (resp. $d^{n}(v) \neq 0$ ). Alors, on a :

$$
d^{m+n}(1)=(m+n) ! \sum_{i=0}^{m+n} \frac{d^{i}(u)}{i !} \frac{d^{m+n-i}(v)}{(m+n-i) !}=\frac{(m+n) !}{m ! n !} d^{m}(u) \cdot d^{n}(v)
$$

Puisque $R$ est un anneau intègre, il suit du (3.9) que $d^{m+n}(1) \neq 0$ et donc $m+n=$ 0 . Il suit que $m=0$. Ceci montre le résultat.

Proposition 3.5. Soit $A$ un monoïde intègre dans $(\mathbf{C}, \otimes, 1)$ et soit $S \subseteq \mathcal{E}(A)$ un sousensemble stable par multiplication. Soit $D: A \longrightarrow A$ une dérivation localement nilpotente sur $A$ et $D_{S}: A_{S} \longrightarrow A_{S}$ son prolongement à $A_{S}$. Alors, si $D_{S}$ est une dérivation localement nilpotente sur $A_{S}$, il suit que pour chaque $s \in S$, on a $\mathcal{E}(D)(s)=0$. Autrement dit, on a $S \subseteq \operatorname{Ker}(\mathcal{E}(D))$.

Proof. On sait que $A$ est un monoïde intègre. Alors, chaque $s \in \mathcal{E}(A)$ est un monomorphisme $s: A \longrightarrow A$. Nous considérons le diagramme commutatif suivant:

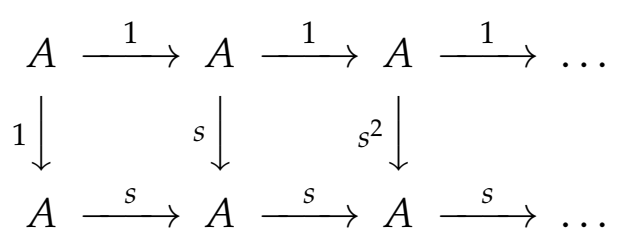

Puisque les colimites filtrantes commutent aux limites finies, en passant à la colimite filtrante dans (3.10), on obtient un monomorphisme $A \longrightarrow A_{s}$. De plus, le sous-ensemble $S$ étant stable par multiplication, la colimite filtrante des monomorphismes $A \longrightarrow A_{S}, s \in S$ nous donne un monomorphisme $j_{S}: A \longrightarrow A_{S}=$ $\underset{s \in S}{\operatorname{colim}} A_{S}$ dans $A-$ Mod. Il suit que:

$$
\begin{aligned}
& \mathcal{E}\left(j_{S}\right): \mathcal{E}(A) \cong \operatorname{Hom}_{A-\operatorname{Mod}}(A, A) \stackrel{\operatorname{Hom}_{A-\operatorname{Mod}}(A,-)}{\longrightarrow} \operatorname{Hom}_{A-\operatorname{Mod}}\left(A, A_{S}\right) \\
& \cong \operatorname{Hom}_{A_{S}-\operatorname{Mod}}\left(A_{S}, A_{S}\right) \cong \mathcal{E}\left(A_{S}\right)
\end{aligned}
$$

est un monomorphisme d'anneaux.

Il résulte de Proposition 3.1 que $\mathcal{E}\left(D_{S}\right)$ est une dérivation localement nilpotente sur $\mathcal{E}\left(A_{S}\right)$. De plus, $\mathbf{C}$ étant une catégorie $k$-linéaire, $\mathcal{E}\left(A_{S}\right)$ est une $k$-algèbre. Alors, chaque $s \in S \subseteq \mathcal{E}(A)$ étant un élément inversible de $\mathcal{E}\left(A_{S}\right)$, il suit du Lemme 3.4 que $\mathcal{E}\left(D_{S}\right)(s)=0$. De plus, il est clair qu'on a un diagramme commutatif:

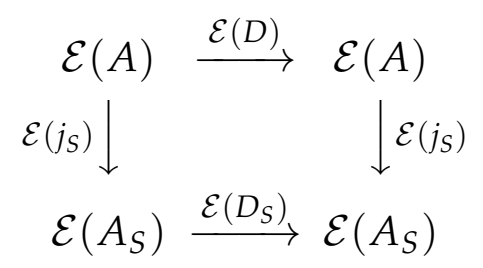

Alors, on a

$$
0=\mathcal{E}\left(D_{S}\right)(s)=\left(\mathcal{E}\left(D_{S}\right) \circ \mathcal{E}\left(j_{S}\right)\right)(s)=\left(\mathcal{E}\left(j_{S}\right) \circ \mathcal{E}(D)\right)(s)
$$

Dans (3.11), on sait que $\mathcal{E}\left(j_{S}\right): \mathcal{E}(A) \longrightarrow \mathcal{E}\left(A_{S}\right)$ est un monomorphisme et donc il suit de (3.12) que $\mathcal{E}(D)(s)=0$. Ceci montre le résultat. 
Inversement, soit $D: A \longrightarrow A$ est une dérivation localement nilpotente et soit $S \subseteq \mathcal{E}(A)$ un sous-ensemble stable par multiplication tel que $S \subseteq \operatorname{Ker}(\mathcal{E}(D))$. Nous montrons que son prolongement $D_{S}: A_{S} \longrightarrow A_{S}$ est une dérivation localement nilpotente.

Proposition 3.6. Soit $A$ un monoïde commutatif et soit $D: A \longrightarrow A$ une dérivation localement nilpotente. Considérons un sous-ensemble $S \subseteq \operatorname{Ker}(\mathcal{E}(D))$ qui est stable par multiplication. Alors, $D_{S}$ est une dérivation localement nilpotente sur $A_{S}$.

Proof. En posant $\mathcal{E}(D)(s)=0$ dans (3.8) pour chaque $s \in S$, on a un diagramme commutatif:

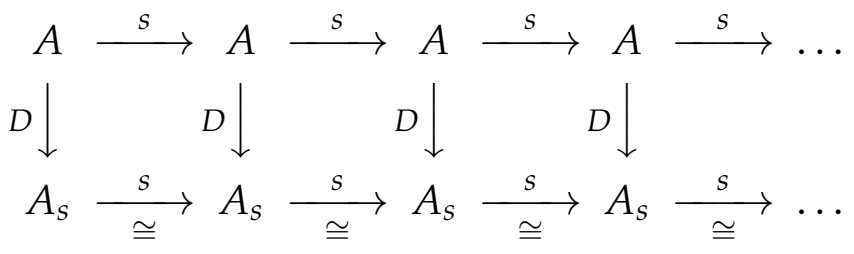

Plus généralement, on a un diagramme commutatif:

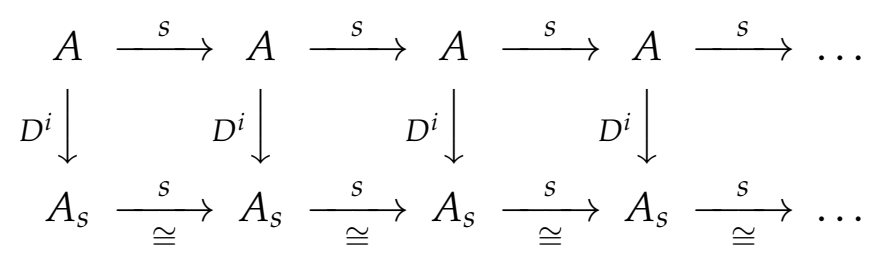

pour chaque $i>0$. Il suit du (3.14) que

$$
\operatorname{Ker}\left(D_{s}^{i}\right)=\operatorname{colim}\left(\operatorname{Ker}\left(D^{i}\right) \stackrel{s}{\longrightarrow} \operatorname{Ker}\left(D^{i}\right) \stackrel{s}{\longrightarrow} \operatorname{Ker}\left(D^{i}\right) \stackrel{s}{\longrightarrow} \ldots\right)
$$

Puisque $D$ est une dérivation localement nilpotente sur $A$, on a donc:

$$
\begin{aligned}
\underset{i \geq 1}{\operatorname{colim}} \operatorname{Ker}\left(D_{s}^{i}\right) & =\operatorname{colim}\left(\operatorname{colim} \operatorname{Ker}\left(D^{i}\right) \stackrel{s}{\longrightarrow} \operatorname{colim} \operatorname{Ker}\left(D^{i}\right) \stackrel{s}{\longrightarrow} \operatorname{colim} \operatorname{Ker}\left(D^{i}\right) \stackrel{s}{\longrightarrow} \ldots\right) \\
& =\operatorname{colim}(A \stackrel{s}{\longrightarrow} A \stackrel{s}{\longrightarrow} A \stackrel{s}{\longrightarrow} \ldots)=A_{s}
\end{aligned}
$$

Enfin, on a:

$$
\underset{i \geq 1}{\operatorname{colim}} \operatorname{Ker}\left(D_{S}^{i}\right)=\underset{s \in S}{\operatorname{colim}} \operatorname{colim} \operatorname{Ker}\left(D_{S}^{i}\right)=\underset{s \in S}{\operatorname{colim}} A_{S}=A_{S}
$$

d'où ce résultat.

\section{References}

[1] A. Banerjee, Centre of monoids, centralizers, and localisation. Comm. Algebra 40, No. 11, 3975-3993 (2012).

[2] A. Banerjee, On the Lie transformation algebra of monoids in symmetric monoidal categories, Rend. Sem. Mat. Univ. Padova, Vol. 131, 151-157 (2014). 
[3] H.-J. Baues, M. Jibladze, A. Tonks, Cohomology of monoids in monoidal catégorie, in: Operads: Proceedings of renaissance conferences. Contemp. Math. 202, 137-165 (1997).

[4] S. M. Bhatwadekar, D. Daigle, On finite generation of kernels of locally nilpotent $R$-derivations of $R[X, Y, Z]$, J. Algebra 322 (2009), 2915-2926.

[5] D. Daigle, On some properties of locally nilpotent derivations, J. Pure Appl. Algebra 114 (1997), 221-230.

[6] D. Daigle, G. Freudenburg, Locally nilpotent derivations over a UFD and an application to rank two locally nilpotent derivations of $k\left[X_{1}, \ldots, X_{n}\right]$, J. Algebra 204 (1998), 353-371.

[7] P. Deligne, Catégories tannakiennes, in: The Grothendieck Festschrift, vol. II, in: Progr. Math., vol. 87, Birkhäuser Boston, Boston, MA, 1990, pp. 111-195.

[8] B. Eckmann, P. J. Hilton, Group-like structures in general categories. I. Multiplications and comultiplications, Math. Ann., 145, 227-255 (1961/62)

[9] A. van den Essen, Locally nilpotent derivations and their applications III, J. Pure Appl. Algebra 98 (1995), 15-23.

[10] G. Freudenburg, Algebraic theory of locally nilpotent derivations. Encyclopaedia of Mathematical Sciences, 136. Invariant Theory and Algebraic Transformation Groups, VII. Springer-Verlag, Berlin, 2006.

[11] M. Hakim, Topos annelés et schémas relatifs, Ergebnisse der Mathematik und ihrer Grenzgebiete, Band 64, Springer-Verlag, Berlin, New York, 1972.

[12] M. Hovey, Cotorsion pairs and model categories. Interactions between homotopy theory and algebra, 277-296, Contemp. Math., 436, Amer. Math. Soc., Providence, RI, 2007.

[13] M. Prest, A. Ralph, Locally finitely presented categories of sheaves of modules, Manchester Institute for Mathematical Sciences Eprint 2010.21 (2010).

[14] B. Toën, M. Vaquié, Au-dessous de $\operatorname{Spec}(\mathbb{Z})$, J. K-Theory 3 (3) (2009) 437-500.

[15] E. M. Vitale, Monoidal categories for Morita theory, Cah. Top. Géom. Diff. Catég, 33 (4) (1992) 331-343.

Institut des Hautes Études Scientifiques,

Le Bois-Marie 35, route de Chartres,

91440, Bures-sur-Yvette, France.

Email: abhishekbanerjee1313@gmail.com 